\title{
Condição de saúde oral e indicativos da síndrome de Burnout em estudantes de Odontologia sedentários e atletas
}

\author{
Adriana Dibo Cruz*; Julia Motta Hatherly**; Leonardo dos Santos Antunes***; Gabriela Alessandra da \\ Cruz Galhardo Camargo****; Marcelo Freitas Aguiar*****
}

* Prof. Adjunto do Departamento de Formação Específica do Instituto de Saúde de Nova Friburgo da Universidade Federal Fluminense

** Estudante de graduação, Bolsista de Iniciação Científica do edital Fopin 2015 da Universidade Federal Fluminense

*** Prof. Adjunto do Departamento de Formação Específica do Instituto de Saúde de Nova Friburgo da Universidade Federal Fluminense

**** Prof. Adjunto do Departamento de Formação Específica do Instituto de Saúde de Nova Friburgo da Universidade Federal Fluminense

***** Prof. Adjunto do Departamento de Formação Específica do Instituto de Saúde de Nova Friburgo da Universidade Federal Fluminense

Recebido em 18/01/2017. Aprovado em 08/04/2017.

\section{RESUMO}

O estudo objetivou comparar a condição de saúde oral e os indicativos de síndrome de Burnout em estudantes de Odontologia sedentários e atletas. Após aprovação do projeto pelo Comitê de Ética em Pesquisa, foi selecionada, por conveniência, uma amostra não probabilística de estudantes do terceiro ano do Curso de Odontologia de uma Universidade Federal brasileira com características semelhantes quanto ao sexo, idade, escolaridade e nível socioeconômico, para compor dois grupos independentes: 1) atletas e 2) sedentários. Para as estimativas da condição de saúde oral foram realizadas avaliação periodontal completa, levantamento de dados de um questionário elaborado a partir de uma ferramenta validada, o Maslach Burnout Inventory-General Survey (MBI), preenchimento de prontuário de paciente e radiografia panorâmica. Os dados coletados foram analisados estatisticamente pelo teste T ou Mann-Whitney, análise de regressão logística simples e correlação de Pearson (significância em 5\%, $\alpha=0,05$ ). Houve variações na condição de saúde oral de estudantes de Odontologia atletas em relação aos sedentários quanto ao sangramento à sondagem, a profundidade de sondagem e achados radiográficos, que apresentaram variações estatisticamente significativas $(\mathrm{p}<0,05)$ com piores resultados para $\mathrm{o}$ grupo de sedentários. O sangramento à sondagem foi o principal indicativo da discrepância que apresentou correlação fraca $(\rho=0,31)$ com a exaustão emocional, que é a medida do sentimento de estar emocionalmente sobrecarregado e esgotado. Os estudantes sedentários são mais sensíveis a problemas e tensões desencadeados no ambiente acadêmico, com maior propensão a instalação de danos somáticos como os problemas orais.

Descritores: Estudantes de Odontologia. Saúde Bucal. Doenças Periodontais. Radiografia Panorâmica. Esgotamento Profissional. 


\section{INTRODUÇÃOO}

O estresse crônico no ambiente ocupacional, de trabalho ou estudo, é um fator de risco para o desenvolvimento da síndrome de Burnout. Essa síndrome caracteriza-se como um esgotamento físico e mental causado principalmente por falha ou por incapacidade de enfrentamento dos problemas e tensões desencadeadas pelas relações ocupacionais e exigências acadêmicas.

A síndrome é multidimensional, constituída por exaustão mental e emocional; dispersonalização com sentimento de apatia e atitudes negativas nas relações humanas e na vida; e baixa realização pessoal associados a uma sensação de frustração e fracasso ${ }^{1}$. O agravamento da síndrome pode desencadear vários problemas físicos de saúde, não somente psicológicos ${ }^{2,3}$.

No campo da Odontologia têm sido observado ${ }^{4}$ que o fator psicológico tem capacidade de induzir modificações no sistema imunológico e no comportamento do indivíduo, que desencadeariam alterações da saúde oral. Apesar do papel exato deste fator não se encontrar totalmente elucidado, há indícios da relação do estresse com alterações da progressão de lesões de cáries, de disfunções da articulação temporomandibular, de problemas periodontais, entre outras ${ }^{5}$. Na periodontia, a relação entre o fator psicológico como causa de desequilíbrio fisiológico e da homeostase, que alteram a resposta imunoinflamatória aos antígenos do biofilme polimicrobiano e desencadeiam os problemas periodontais, já vem sendo estudado há muitos $a_{n o s}^{4}$. E o estresse vem sendo considerado como um possível fator de risco para a doença periodontal ${ }^{2,6,7}$.

Alguns estudos ${ }^{2}$ apontam que as atividades físicas aeróbicas são capazes de diminuir o estresse, melhorar o sistema cardiovascular e as respostas inflamatórias, entre outras. Foi observado $^{6}$ também que os exercícios físicos podem ter um efeito supressivo nas doenças periodontais, pois melhorariam o fluxo sanguíneo periférico no tecido periodontal local. Também foi observado ${ }^{8}$ que adolescentes atletas tinham melhor saúde oral que os sedentários.

A detecção precoce da presença de indicativos desta síndrome, em populações específicas, pode ajudar a implementação de medidas preventivas e estratégias de enfrentamentos de problemas e tensões dos diferentes contextos ocupacionais e acadêmicos, não permitindo que ocorra a instalação de danos somáticos como os problemas orais. Assim, o objetivo do presente estudo foi avaliar se existem variações na condição de saúde oral e nos indicativos de síndrome de Burnout de estudantes de Odontologia atletas e sedentários.

\section{MATERIAIS E MÉTODOS}

O estudo foi iniciado após aprovação do projeto (CAAE: 37420614.0.0000.5626) pelo comitê de ética em pesquisa humana em 18/5/2015, número do comprovante: 093028/ 2014.

Foi selecionada por conveniência uma amostra não probabilística de estudantes do terceiro ano do Curso de Odontologia de uma Universidade Federal brasileira com características semelhantes quanto ao sexo, idade, escolaridade e nível socioeconômico, para compor dois grupos independentes: Grupo de Atletas, composto por estudantes de Odontologia que informaram realizar atividades físicas frequentemente e ininterruptamente, $\mathrm{c}$ pelo menos 1 ano; e Grupo de Sedentários, composto por estudantes de Odontologia que informaram não realizar nenhum tipo de atividade física, mesmo 
que eventualmente, há pelo menos 1 ano.

A amostra foi composta por 40 estudantes ( $\mathrm{N}=20$ para cada grupo), não vulneráveis e sem conflito de interesses, que concordaram e se predispuseram a participar deste estudo, como participante da pesquisa, assinando o Termo de Consentimento Livre e Esclarecido.

Os participantes do estudo foram submetidos à avaliação periodontal completa e também preencheram um questionário elaborado a partir de uma ferramenta validada ${ }^{1,9,10}$, o Maslach Burnout Inventory-General Survey (MBI), para investigação de indicativos da síndrome de Burnout. Na sequência, os participantes do estudo receberam atendimento odontológico, de acordo com suas necessidades. Para o plano de tratamento foram realizados exame físico, com preenchimento de prontuário, e uma radiografia panorâmica inicial, seguindo o protocolo institucional. Assim, para o estudo foram coletados dados de interesse relacionados ao perfil sociodemográfico do participante da pesquisa, da radiografia panorâmica, da avaliação periodontal e do questionário da pesquisa para investigação de indicativos da síndrome de Burnout.

A avaliação periodontal foi realizada por um único avaliador treinado e calibrado, em estudo piloto (kappa=0,93), que com uso de uma sonda periodontal milimetrada aferiu 6 sítios periodontais (mesiovestibular, vestibular, distovestibular, mésio-lingual/palatino, lingual/ palatino e disto-lingual/palatino) de cada elemento dentário de modo independente, sendo coletados os dados de profundidade de sondagem (distância em mm entre a margem gengival e o fundo do sulco gengival), recessão gengival (distância em $\mathrm{mm}$ entre a junção cemento esmalte e a margem gengival), sangramento à sondagem (observado durante a mensuração de profundidade de sondagem). A presença de cálculo foi avaliada por inspeção visual, marcando os sítios que possuíam placa visível. Todos os dados foram marcados no periograma, tabulados e computada a média por elemento. As médias de cada medida foram utilizadas para a análise estatística.

A avaliação da radiografia panorâmica foi realizada por um único avaliador radiologista experiente, que teve possibilidade de utilizar todos os recursos necessários para melhorar a qualidade do laudo, além de solicitar exames radiográficos intraorais adicionais quando necessário. Não houve restrições de tempo para avaliação, que foi quantitativa, classificando os achados radiográficos, de cada órgão dentário independentemente, seguindo os escores: 0 - Para dente ausente ou perdido; 1 - para dente hígido; 2 - para dente com contenção de tratamento ortodôntico e reabsorção radicular externa, 3 - para dente não irrompido ou incluso/impactado; 4 - para dente com lesão de cárie; 5 - para dente com restauração sem lesão de cárie recorrente, 6 - para dente com restauração e com lesão de cárie recorrente; 7 para dente com tratamento endodôntico; 8 - para outros achados de reabilitação oral como próteses fixas e implantes. A amostra não possuía nenhum outro tipo de achado radiográfico além dos citados no escore utilizado. Desvios de normalidade ou alterações de desenvolvimento não foram consideradas na análise. Os escores foram somados para a análise estatística.

A avaliação do questionário para investigação de indicativos da síndrome de Burnout seguiu o roteiro de avaliação fornecido pelo Mind Garden, Inc., o MBI: Instruments and Scoring Guides, na compra da licença de uso do MBI. O instrumento consiste de 22 questões que se subdividem em três subescalas: Exaustão Emocional com 9 questões $(1,2,3,6,8,13,14,16$ 
e 20), para a Despersonalização com 5 questões (5, $10,11,15$ e 22) e para a Baixa Realização Pessoal com 8 questões $(4,7,9,12,17,18,19$ e 21$)$. Todas as questões são avaliadas em escala ordinal de Likert com 7 pontos, variando de 0 (nunca) a 6 (sempre). Os dados foram somados de acordo com a subscala considerada e utilizados para a análise estatística.

Todos os dados foram tabulados e realizou a análise exploratória de normalidade utilizando o teste de Shapiro-Wilk. Os dados foram analisados utilizado teste T de Student ou Mann-Whitney. As variáveis que apresentaram significância estatística foram analisadas pela análise de regressão logística simples. Já a correlação de Pearson foi utilizada para avaliar a relação da condição de saúde oral, independentes dos grupos, com os indicativos da síndrome de Burnout. Todas as análises estatísticas foram conduzidas com nível de significância em $5 \%(\alpha=0,05)$.

\section{RESULTADOS}

$\mathrm{Na}$ tabela 1 é possível observar a homogeneidade das variáveis idade, sexo, exaustão emocional, despersonalização, realização pessoal, índice de placa e recessão gengival dos grupos de estudantes sedentários e atletas que não apresentaram diferenças estatísticas entre eles ( $\mathrm{p}>0,05)$. Já as variáveis sangramento à sondagem, profundidade de sondagem e achados radiográficos apresentaram variações estatisticamente significativas $(p<0,05)$ entre os grupos sedentário e atletas, tendo os alunos do grupo de sedentários apresentado os piores resultados.

Tabela 1 - Valores de médias e desvios padrão dos grupos sedentários e atletas

\begin{tabular}{|c|c|c|c|c|}
\hline Variáveis & Sedentários & Atletas & $\begin{array}{l}\text { Resultados } \\
\text { estatístico }\end{array}$ & Valor de $p$ \\
\hline Idade & $21,90(1,89)$ & $21,55(1,36)$ & $z=0,0947$ & 0,9246 \\
\hline $\mathrm{Sexo}^{\#}$ & $1,15(0,37)$ & $1,40(0,50)$ & $\mathrm{z}=1,3525$ & 0,1762 \\
\hline Exaustão Emocional & $21,75(11,42)$ & $19,00(9,39)$ & $\mathrm{t}=0,8318$ & 0,4107 \\
\hline Despersonalização & $6,80(7,90)$ & $4,60(5,31)$ & $\mathrm{z}=0,7574$ & 0,4488 \\
\hline Realização pessoal & $31,10(7,52)$ & $34,50(6,05)$ & $t=-1,5759$ & 0,1232 \\
\hline Índice de placa & $5,23(7,97)$ & $1,55(2,52)$ & $\mathrm{z}=1,9341$ & 0,0531 \\
\hline $\begin{array}{l}\text { Sangramento a } \\
\text { sondagem }\end{array}$ & $1,47(1,44)$ & $0,61(0,84)$ & $\mathrm{z}=2,0152$ & $0,0439 *$ \\
\hline $\begin{array}{l}\text { Profundidade de } \\
\text { sondagem }\end{array}$ & $1,57(0,24)$ & $1,42(0,16)$ & $\mathrm{t}=2,2030$ & $0,0337 *$ \\
\hline Recessão gengival & $0,90(0,86)$ & $0,78(0,90)$ & $\mathrm{z}=0,4058$ & 0,6849 \\
\hline Achados radiográficos & $53,55(18,81)$ & $42,35(9,37)$ & $\mathrm{z}=2,0829$ & $0,0373 *$ \\
\hline
\end{tabular}

Dentre as variáveis que apresentaram sangramento à sondagem e os achados significância estatística (tabela 2), a chance de radiográficos podem ser encontrados com uma 
frequência maior, aproximadamente 0,5 vezes e 1 vez maior, respectivamente, em grupos sedentários do que no grupo de atletas.

As frequências de dimensões de Burnout em estudantes de Odontologia considerando os grupos sedentário e atletas são exibidas na figura 1 e a correlação das variáveis utilizadas para avaliar a condição de saúde oral, independentemente do grupo, em relação aos indicativos da síndrome de Burnout, é demonstrada na tabela 3, observando que houve uma correlação fraca entre exaustão emocional e sangramento à sondagem $(\rho=0,31)$. As demais variáveis apresentaram correlação desprezível $^{11}$.

Tabela 2 - Análise de regressão logística simples sobre a relação das variáveis independentes significativas sobre o hábito de prática esportiva.

Odds Std. $\quad$ Valor- $\quad$ IC 95\%

\begin{tabular}{lcccccc} 
Variáveis & Logit Pi & Ratio & Err. & $\mathrm{Z}$ & $\mathrm{P}$ & \\
\hline $\begin{array}{l}\text { Sangramento à } \\
\text { sondagem }\end{array}$ & $\mathrm{Y}=0,6816-(0,704 \mathrm{X} 1)$ & 0,495 & 0,170 & $-2,050$ & 0,040 & 0,252 a 0,970 \\
$\begin{array}{l}\text { Profundidade de } \\
\text { sondagem }\end{array}$ & $\mathrm{Y}=5,6613-(3,813 \mathrm{X} 1)$ & 0,022 & 0,042 & $-2,000$ & 0,046 & 0,001 a 0,930 \\
$\begin{array}{l}\text { Achados } \\
\text { radiográficos }\end{array}$ & $\mathrm{Y}=2,9034-(0,062 \mathrm{X} 1)$ & 0,940 & 0,028 & $-2,110$ & 0,035 & 0,888 a 0,996 \\
\hline
\end{tabular}

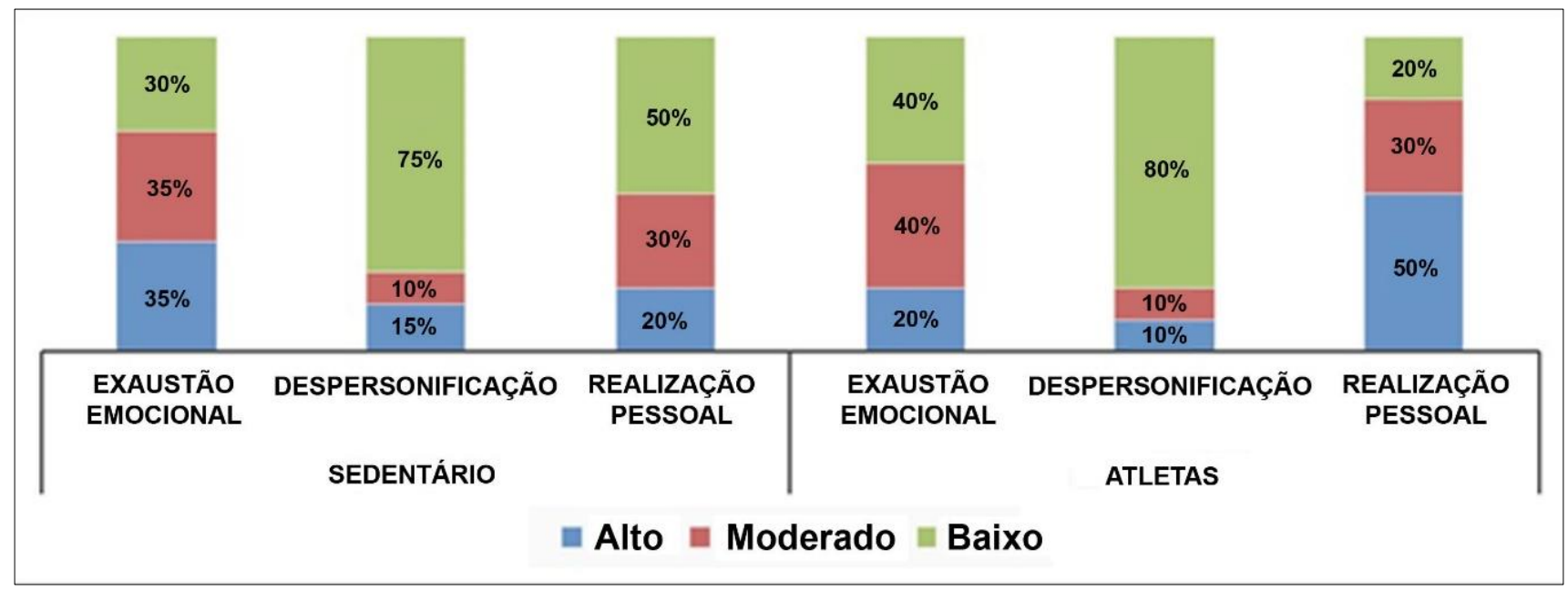

Figura 1 - Frequência de dimensões de Burnout considerando o hábito de prática esportiva. - A interpretação das escalas Exaustão emocional e Despersonificação segue direção oposta à da escala Realização pessoal 
Tabela 3 - Correlação da condição de saúde oral, independente do grupo, com os indicativos da síndrome de Burnout

\begin{tabular}{lccc}
\hline & Exaustão Emocional & Despersonalização & Realização pessoal \\
\hline $\begin{array}{l}\text { Sangramento à } \\
\text { sondagem }\end{array}$ & $0,31^{*}$ & 0,17 & 0,15 \\
$\begin{array}{l}\text { Profundidade de } \\
\text { sondagem }\end{array}$ & 0,09 & $-0,06$ & 0,18 \\
$\begin{array}{l}\text { Achados } \\
\text { Radiográficos }\end{array}$ & 0,11 & $-0,22$ & 0,16 \\
\hline * 0,3 a 0,5 positivo ou negativo indica uma correlação fraca. \\
0 a 0,3 positivo ou negativo indica uma correlação desprezível. ${ }^{11}$
\end{tabular}

\section{DISCUSSÃO}

O presente estudo comparou a condição de saúde oral e indicativos de síndrome de Burnout de dois grupos de estudantes de Odontologia distintos quanto ao hábito da prática esportiva. Todas as comparações se deram para avaliar se os atletas possuíam diferenças em algum parâmetro de avaliação em relação aos sedentários. Para tanto, a seleção da amostra conveniente representativa foi o grande desafio do estudo, uma vez que poderia representar um viés de seleção ${ }^{12}$. Ambos os grupos estudados deveriam ser o mais homogêneo e pareados, quanto possível, em relação as variáveis sexo, idade, escolaridade e nível socioeconômico, que são modificadores de efeito e poderiam interferir diretamente nos resultados do estudo ${ }^{3,12-}$ 15. Mas, principalmente, deveriam atender aos critérios de inclusão de grupo, que foram realizar atividades físicas frequentes e ininterruptas ${ }^{16}$ há pelo menos 1 ano, para o grupo de atletas, e não realizar nenhum tipo de atividade física, mesmo que eventualmente, há pelo menos 1 ano, para o grupo de sedentários.

A grande maioria ( $\approx 66 \%$ ) da população-alvo disponível praticava, eventualmente, algum tipo de esporte, geralmente esportes individuais em academias, sendo classificadas como atividades físicas sazonais ${ }^{16}$, e ainda, com grandes intervalos de descontinuação, devido aos períodos de provas e férias escolares e, portanto, não poderiam compor a amostra em nenhum grupo. Assim, população real foi bem limitada, sendo excluídos apenas 3 indivíduos do total, que poderiam compor a amostra no grupo de sedentários, por discrepância de idade. Não houve nenhum caso de perda por desistência em participar do estudo ou recusa. Com isso, os grupos não foram rigorosamente pareados, porém não apresentaram diferenças estatisticamente significativas das variáveis idade e sexo.

A frequência de indicativos de síndrome de Burnout foi maior entre os estudantes de Odontologia mais velhos, casados e em fases mais avançadas do curso $^{14}$. Foi observada ${ }^{12}$ relação significativa entre o ano do curso de odontologia e a dimensão exaustão emocional, indicando que estudantes em fases iniciais do curso teriam uma maior dificuldade de adaptação com o novo ambiente, estando vulneráveis a frustrações e tendo pouca realização pessoal, enquanto que os estudantes de fases mais avançadas, devido as exigências crescentes do curso, teriam uma maior exaustão emocional. No presente estudo o nível de escolaridade e socioeconômico eram equivalentes, 
uma vez que todos os participantes do estudo eram provenientes de uma mesma instituição de ensino, todos do terceiro ano do curso de Odontologia, cursando o primeiro curso superior, não eram casados, e estavam tendo a primeira experiência de morar longe dos familiares. Em estudo ${ }^{17}$ que possuía amostra melhor distribuída em relação a variável sexo, foram encontrados maiores indicativos da síndrome de Burnout nos participantes do sexo masculino. Mas o mesmo não se confirmou em outro estudo ${ }^{12}$, que não identificou nenhuma diferença relacionada ao sexo. No presente estudo não foi feita tal comparação pois haviam poucos participantes $(\mathrm{N}=11 ; 27,5 \%)$ do sexo masculino, com o grupo de atletas tendo 8 (40\%) participantes e o grupo de sedentários $3(15 \%)$ participantes.

$\mathrm{Na}$ literatura ${ }^{3,9,12,14-15,17-19}$ é relatada uma frequência maior de casos com altos indicativos de síndrome de Burnout em estudantes de Odontologia do que de outros cursos. No estudo de Campos et al. $(2012)^{12}$, que relata a realidade brasileira de estudantes de Odontologia, foi observada a frequência de $17 \%$ de casos com indicativos da presença da síndrome. No estudo de Atalayin et al. $(2015)^{15}$, com estudantes de Odontologia da Turquia, observou-se que 22,3\% apresentavam altos indicativos de exaustão emocional, $16,7 \%$ de despersonalização e 17,9\% de baixa realização pessoal. No presente estudo, os grupos não diferiram estatisticamente entre si em relação aos indicativos da síndrome de Burnout, que se apresentaram altos e compatíveis, na média, com os valores encontrados nos estudos citados $^{12,15}$. Dos participantes do atual estudo 27,5\% apresentavam altos indicativos de exaustão emocional, $12,5 \%$ de despersonalização e $35 \%$ de baixa realização pessoal, com o grupo de sedentários apresentando os maiores indicativos para presença desta síndrome (figura 1).

Já em relação a condição de saúde oral dos participantes foram observadas diferenças estatisticamente significativas das variáveis sangramento à sondagem, profundidade de sondagem e achados radiográficos, que se mostraram piores no grupo de sedentários. Oliveira et al. $(2015)^{7}$ indicaram que a doença periodontal poderia ser considerada um indicador de risco de má aptidão física, principalmente em homens. Foi sugerido ${ }^{6}$ haver efeitos interativos entre obesidade e aptidão física com o estado de saúde periodontal. Além de estreita relação ${ }^{2}$ entre saúde bucal com as condições gerais saúde e aptidão física. Goyal et al. $(2013)^{4}$ relataram que desde os anos 1950, as doenças periodontais estão sendo relacionadas aos fatores emocionais. $\mathrm{O}$ estresse psicológico poderia regular a resposta imune e perturba a homeostase por meio de uma rede complexa de sinais que ligam os sistemas nervoso, endócrino e imunológico. Mas a associação direta entre doença periodontal e estresse ainda não foi comprovada, por limitações dos modelos animais, dificuldade para quantificar o estresse, além das múltiplas variáveis que podem afetam a gravidade da doença periodontal clinicamente.

Muito do observado no presente estudo poderia ser explicado pelos resultados do estudo de Anttonen et al. $(2015)^{8}$, que indicaram que o comportamento de adolescentes atletas, principalmente do sexo feminino, em relação aos cuidados com a saúde oral era mais favorável do que a dos adolescentes não atletas. Com isso a melhor condição de saúde oral dos atletas seria a consequência de um maior cuidado e motivação geral com a própria saúde. No presente estudo, devido ao fato de ter encontrado diferenças significativas dos achados radiográficos entre os 
grupos, e os achados serem consideradas, principalmente, alterações adquiridas de longo prazo, pode ser especulado que o esporte em si não contribuiu para a melhor saúde oral de atletas, mas indicaria quais as pessoas que possuem atitudes mais favoráveis em relação a própria saúde bucal. Além disso, como houve correlação positiva fraca entre o sangramento à sondagem com a exaustão emocional, que seria o primeiro indicativo da síndrome de Burnout, pode-se especular ainda que essas pessoas que praticam esporte possuem melhores condições de enfrentamento de problemas emocionais desencadeados das exigências das relações acadêmicas ${ }^{19}$, sem, contudo, fazer nenhuma inferência de causalidade.

Embora se tenha observado indícios de relação entre a prática de atividades esportivas e melhor saúde oral não é possível fazer extrapolações dos resultados do presente estudo, pois, houve uma grande limitação imposta pela casuística. Porém, devido ao fato de as amostras não serem probabilísticas, tendo grande controle de variáveis, é possível traçar estratégias de atenção e implementação de medidas preventivas, tanto de saúde oral como para enfrentamentos de problemas e tensões psicológicas, neste contexto acadêmico específico, para o grupo de estudantes sedentários, que estariam mais propensos a desenvolver problemas somáticos.

\section{CONCLUSÃO}

De acordo com os resultados encontrados no presente estudo pode-se concluir que houve variações na condição de saúde oral de estudantes de Odontologia atletas em relação aos sedentários. $\mathrm{O}$ sangramento à sondagem foi o principal indicativo da discrepância na condição de saúde oral dos grupos avaliados, possuindo ainda correlação, mesmo que fraca, com a exaustão emocional. Apesar de não haver diferenças significativas entre os indicativos de síndrome de Burnout nos dois grupos avaliados, o grupo de atletas apresentou os melhores valores em todas as dimensões de Burnout, e com isso, pode-se sugerir que os estudantes sedentários são mais sensíveis a problemas e tensões desencadeados no ambiente acadêmicos e possuem maior propensão a instalação de danos somáticos como os problemas orais.

\section{AGRADECIMENTOS}

À Proppi/UFF pelo auxílio financeiro por meio do edital FOPIN2015.

\section{ABSTRACT Condition of oral health and Burnout syndrome indicatives in sedentary and athlete Dental students}

The study aimed to compare the oral health condition and the Burnout syndrome indicatives of athete and sedentary dental students. After the approval of the project by the research ethics committee a non-probabilistic sample of convenience, with students of third year of dentistry course of a Brazilian Federal University with similar characteristics in relation to sex, age, schooling and socioeconomic level were selected. They composed two independent groups: 1) Atheletes and 2) Sedentaries. For health estimations were performed a periodontal evaluation and data collections from a questionnaire on the basis of a validated tool, the Maslach Burnout Inventory-General Survey (MBI), a medical records and a panoramic radiograph. All data were statistically analyzed by T-test, Mann-Whitney test, simple logistic regression analysis and Pearson's correlation (significance at $5 \%, \alpha=0.05$ ). There were variations in the oral health condition between the athelete and sedentary dental students for bleeding on probe, probe depth and radiographic findings 
that statistically differed $(\mathrm{p}<0.05)$ showing worst results for the sedentary group. The bleeding on probe was the main indicator of the discrepancy and presented a weak correlation $(\rho=0.31)$ with an emotional exhaustion, which measures feelings of being emotionally overextended and exhausted. Sedentary dental students are more sensitive to problems and tensions triggered in the academic environment with a greater propensity to instalation of somatic injuries such as oral problems.

Descriptors: Dental Students. Oral Health. Periodontal Diseases. Panoramic

Radiography. Professional Burnout.

\section{REFERÊNCIAS}

1. Schaufeli WB, Leiter MP, Maslach C, Jackson SE. Maslach Burnout InventoryGeneral Survey. 1996. [Acesso em 07 abr 2017]. Disponível em: http://www. mindgarden.com/products/mbi.htm.

2. Wakai K, Kawamura T, Umemura O, Hara Y, Machida J, Anno T, et al. Associations of medical status and physical fitness with periodontal disease. J Clin Periodontol. 1999;26(10):664-72.

3. Galan F, Rios-Santos JV, Polo J, RiosCarrasco B, Bullon P. Burnout, depression and suicidal ideation in dental students. Med Oral Patol Oral Cir Bucal. 2014;19(3):e20611.

4. Goyal S, Gupta G, Thomas B, Bhat KM, Bhat GS. Stress and periodontal disease: The link and logic!! Ind Psychiatry J. 2013;22(1):4-11.

5. Gay-Escoda C, Vieira-Duarte-Pereira DM, Ardevol J, Pruna R, Fernandez J, ValmasedaCastellon E. Study of the effect of oral health on physical condition of professional soccer players of the Football Club Barcelona. Med Oral Patol Oral Cir Bucal. 2011;16(3):e436-9
6. Shimazaki Y, Egami Y, Matsubara T, Koike G, Akifusa S, Jingu S, et al. Relationship Between Obesity and Physical Fitness and Periodontitis. J Periodontol. 2010;81(8):112431.

7. Oliveira JA, Hoppe CB, Gomes MS, Grecca FS, Haas AN. Periodontal disease as a risk indicator for poor physical fitness: a crosssectional observational study. J Periodontol. 2015;86(1):44-52.

8. Anttonen $\mathrm{V}$, Kemppainen A, Niinimaa A, Pesonen P, Tjäderhane L, Jaana L. Dietary and oral hygiene habits of active athletes and adolescents attending ordinary junior high schools. Int J Paediatr Dent. 2014;24(5):35866.

9. Montero-Marin J, Monticelli F, Casas M, Roman A, Tomas I, Gili M, et al. Burnout syndrome among dental students: a short version of the "Burnout Clinical Subtype Questionnaire" adapted for students (BCSQ12-SS). BMC Med Educ. 2011;11:103.

10. Montiel-Company JM, Subirats-Roig C, Flores-Marti P, Bellot-Arcis C, AlmerichSilla JM. Validation of the Maslach Burnout Inventory-Human Services Survey for Estimating Burnout in Dental Students. J Dent Educ. 2016;80(11):1368-75.

11. Mukaka MM. Statistics corner: A guide to appropriate use of correlation coefficient in medical research. Malawi Med J. 2012;24(3):69-71.

12. Campos JA, Jordani PC, Zucoloto ML, Bonafe FS, Maroco J. Burnout syndrome among dental students. Rev Bras Epidemiol. 2012;15(1):155-65.

13. Pau A, Rowland ML, Naidoo S, AbdulKadir R, Makrynika E, Moraru R, et al. Emotional intelligence and perceived stress in dental 
undergraduates: a multinational survey. J Dent Educ. 2007;71(2):197-204.

14. Mafla AC, Villa-Torres L, Polychronopoulou A, Polanco H, Moreno-Juvinao V, ParraGalvis D, et al. Burnout prevalence and correlates amongst Colombian dental students: the STRESSCODE study. Eur J Dent Educ. 2015;19(4):242-50.

15. Atalayin C, Balkis M, Tezel H, Onal B, Kayrak G. The prevalence and consequences of burnout on a group of preclinical dental students. Eur J Dent. 2015;9(3):356-63.

16. Atkin AJ, Sharp SJ, Harrison F, Brage S, Van Sluijs EM. Seasonal Variation in Children's Physical Activity and Sedentary Time. Med Sci Sports Exerc. 2016;48(3):449-56.

17. Alemany Martinez A, Berini Aytes L, Gay Escoda C. The burnout syndrome and associated personality disturbances. The study in three graduate programs in Dentistry at the University of Barcelona. Med Oral Patol Oral Cir Bucal. 2008;13(7):E444-50.
18. Prinz P, Hertrich K, Hirschfelder U, de Zwaan M. Burnout, depression and depersonalisation - psychological factors and coping strategies in dental and medical students. GMS Z Med Ausbild. 2012;29(1):Doc10.

19. Montero-Marin J, Piva Demarzo MM, Stapinsk L, Gili M, Garcia-Campayo J. Perceived stress latent factors and the burnout subtypes: a structural model in dental students. PloS One. 2014;9(6):e99765.

Correspondência para:

Adriana Dibo da Cruz

e-mail: adrianadibo@id.uff.br

Faculdade de Odontologia da UFF

Instituto de Saúde de Nova Friburgo

Rua Dr. Silvio Henrique Braune, 22,

28625-650 Nova Friburgo/RJ 